\title{
Technicality of Managing Cut Throat Injury
}

\author{
Shreyas S. Joshi, Mohan Jagade, Sunil Nichalani, Sunita Bage, Saurabh Agarwal, Neha Pangam \\ Department of Ear, Nose \& Throat, Grant Medical College, Mumbai, India \\ Email: ssj19july@yahoo.co.in
}

Received September 26, 2012; received October 25, 2012; accepted November 12, 2012

\begin{abstract}
Cervical tracheal injuries are relatively uncommon and are frequently associated with esophageal, vascular or spinal injuries. We hereby report a case of cut throat injury with complete transection of trachea and its management.
\end{abstract}

Keywords: Cut Throat; Homicidal; Tracheal Transection

\section{Introduction}

Patients with cut throat injury may present with airway compromise, aspiration, acute blood loss with hypoxemia because of injury to airway and major vessels. These injuries may be accidental, suicidal or homicidal. This is a case report of homicidal cut throat injury using kitchen knife. All the cervical structures in path of knife starting from skin to trachea were severed. Had the carotid artery and internal jugular vein not been narrowly spared, patient would not have survived to reach hospital.

\section{Case Report}

A 45 years old female was admitted to Department of ENT \& Head and neck surgery, JJ Hospital, Mumbai, with homicidal attack by slashing her throat with kitchen knife. On admission she was in shock with pulse 124/min and Blood pressure of 90/60 mm of Hg, respiration shallow and rapid. Air blast was present from cut open trachea. Immediate resuscitation was started after inserting cuffed tracheostomy tube through lower cut end of trachea to secure airway. After which emergency exploration was done under general anaesthesia.

At exploration, horizontal contused lacerated wound of approximately $10 \mathrm{~cm}$ was seen below the level of cricoid extending from anterior border of one sternocleidomastoid to other, falling under zone I of Roon and Christensen classifications [1]. It was cutting through all infrahyoid muscles, medial half of sternomastoid muscles, both lobes of thyroid gland transversely and completely transecting intertracheal membrane between 1st and 2nd tracheal rings. The carotids, internal jugular veins and esophagus were narrowly missed. Thorough betadine, hydrogen peroxide washes given, haemostasis was achieved and a new trachestoma was created at 2nd/3rd tracheal rings.
The transected trachea was sutured circumferentially with interrupted 4-0 Prolene sutures placed at an interval of 3 - $4 \mathrm{~mm}$. Posterior sutures were taken through mucoperichondrium and rest of circumfential trachea sutured perichondrium to perichondruim externally. Cut ends of infrahyoid muscles were sutured. Her postoperative recovery was uneventful. Strict neck flexion was maintained for 10 days with help of customized neck collar. Fibreoptic laryngoscopy and bronchoscope was done on 21st post operative day. The larynx was found to be normal. A thin neat scar can be seen at the sutured site without any stenosis (Figures 1 and 2). Weaning of tracheostomy tube was started from 24th post operative day and decannulated on 28th post operative day without any respiratory difficulty. After five weeks of her life threatening injury, she was discharged from hospital.

\section{Discussion}

In case of cut throat or penetrating injuries of neck, one should keep in mind the possibility of following vital structures being injured like larynx, pharynx, trachea, oesophagus, major neck vessels, nerve roots and spinal cord.

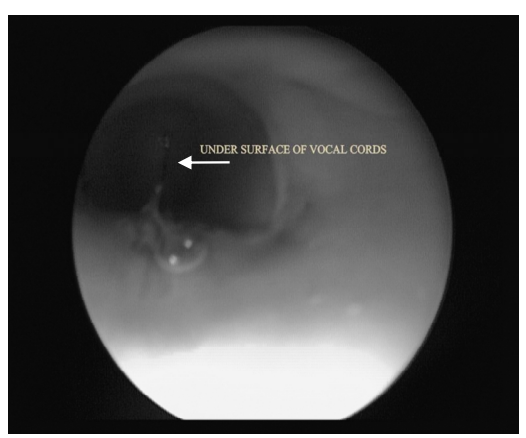

Figure 1. Post operative tracheoscopy done by flexible laryngoscope from tracheostoma (white arrow showing undersurface of vocal cords). 


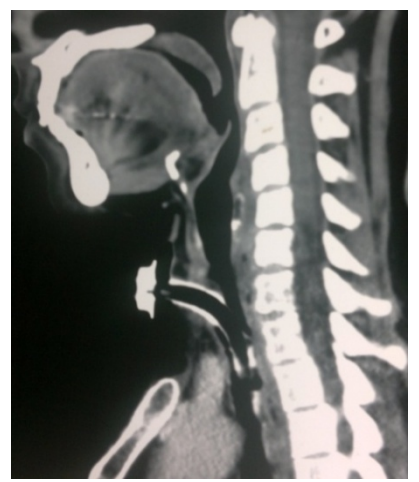

Figure 2. CT neck showing patent tracheal lumen.

Clinical examination and investigations should aim towards detecting any injury to these structures. Investigative procedures should include CT scan, x-ray of the neck \& chest, barium swallow, direct laryngoscopy, bronchoscopy.

Bubbling of air through neck wound indicates perforation of larynx or trachea. The presence of retropharyngeal air seen in lateral view of x-ray neck indicates perforation of either the pharynx or oesophagus. Le May [2] in a study of 25 cases of penetrating wound of neck in Vietnam war found the presence of retro-pharyngeal air a good indication of perforation of perforation of either the pharynx or oesophagus, which may also be demostrated in barium swallow. However, negative result does not rule out possibility of a perforation.

In the reanastomosis of a transected trachea, most authors advocate the use of absorbable suture. Bryce [3] uses one or two stainless steel wires in addition to absorbable suture to provide strength. He keeps head of patient in flexed position post-operatively for 7 to 10 days by means of a suture from the chin to the sternum to relieve tension on the anastomosis. However we sutured cut end of trachea with non absorbable prolene 3-0 extramucosal intermittent sutures. We kept neck in flexion for first 10 days with customised neck collar. The aver- age length of trachea as determined by cadevric studies is $11 \mathrm{~cm}$. Lowering of trachea by suprathroid attachments $(2-3.5 \mathrm{~cm})$ [4]; flexion of neck $(4-5 \mathrm{~cm})$ mobilzation of right hilum with divison of pulmonary ligament (3.5 - 5 $\mathrm{cm}$ ); division of left main stem bronchus with reinsertion into bronchius intermeduis; freeing the pulmonary vessels from pericordium; an advancement of cervical trachea on a vascular pedicle from inferior thyroid artery (4 $\mathrm{cm}$ ) have all been described [5].

\section{Conclusion}

Thus in a case of transected trachea securing airway by doing trachestomy below the injury and just anastomosing and maintaning neck in flexion is all that is required to permit tension free anastomosis. This provides patent non compromised airway with relatively simple surgical technique which can be done even in peripheral hospitals.

\section{REFERENCES}

[1] J. J. Fagan and A. J. Nicol, "Neck Trauma, Scott Browns Torhinolaryngology and Head and Neck Surgery," 7th edition, Vol. 2, Hodder Arnold, London, pp. 1766-1776.

[2] S. R. Le May, "Penetrating Wounds of the Larynx and Cervical Trachea,” Archives of Otolaryngology, Vol. 94, No. 6, 1971, pp. 558-565. doi:10.1001/archotol.1971.00770070858011

[3] D. P. Bryce, “The Surgical Management Laryngotracheal Injury,” The Journal of Laryngology \& Otology, Vol. 86, No. 6, 1972, pp. 547-587. doi:10.1017/S0022215100075605

[4] H. H. Dedo and N. H. Fishman, "Laryngeal Release and Sleeve Resection for Ttracheal Stenosis,” Annals of Otology, Rhinology and Laryngology, Vol. 78, No. 2, 1969, pp. 285-295.

[5] D. F. Knudsen and R. Cohn, "Tracheal Stenosis,” California Medicine, Vol. 115, No. 3, 1971, pp. 1-6. 